\title{
Calorimetric Study Coupled With A Numerical Optimization Technique For The Ca-Sb System
}

\author{
A. Bouhajib ${ }^{1,2}$, M. Notin ${ }^{1}$, A. Nadiri ${ }^{2}$ and A. Yacoubi ${ }^{2}$ \\ 'Laboratoire de Thermodynamique Métallurgique, Faculté des Sciences Université de Nancy I \\ B.P. 239-54506 VANDOEUVRE-les-NANCY Cedex-FRANCE \\ ${ }^{2}$ Equipe de Sciences des Matériaux, Département de Chimie, Faculté des Sciences \\ Meknès B.P. 4010-MAROC.
}

\begin{abstract}
The enthalpies of formation of liquid alloys and intermetallic compounds of the (Ca-Sb) system have been measured by direct reaction calorimetry (drop method). The phase relations are discussed from the results of differential thermal analysis (DTA) coupled with a numerical optimisation technique.
\end{abstract}

\section{INTRODUCTION}

The Ca-Sb system presents several compounds without solubility domains. Only primary solution shows the extended composition. Intermediate compounds are formed by very exothermic reactions. Fundamental information of phases detected in the $\mathrm{Ca}-\mathrm{Sb}$ system are summarised in Table 1.
- The primary $\beta$-calcium phase contains up to $3.8 \%$ $\mathrm{Sb}$ at eutectic temperature $\left(650^{\circ} \mathrm{C}\right)$ following Niyazova, Vakhobov and Dzhuraev /1/.

- The calcium-rich phase is probably $\mathrm{Ca}_{2} \mathrm{Sb}$, following Eisenmann and Schäfer $/ 2 /$.

- The $\mathrm{Ca}_{5} \mathrm{Sb}_{3}$ phase was characterised by Martinez-Rippoll and Brauer $B /$ and seems to be the compound identified as $\mathrm{Ca}_{7} \mathrm{Sb}_{4}$ by Brauer and Muller /4/ or as $\mathrm{Ca}_{3} \mathrm{Sb}_{2}$ by Niyazova et al. $/ 1 /$.

- The median compound is $\mathrm{Ca}_{11} \mathrm{Sb}_{10}$; its structure and the formula were established by Deller and Eisenmann 15/. This corresponds to a compound presented by Niyazova et al. /1/ as CaSb.

- The antimony-rich compound is $\mathrm{CaSb}_{2}$ as proposed by Deller et al. $15 /$, instead of $\mathrm{CaSb}_{3}$ as suggested by Niyazova et al. /1/.

- The primary antimony solution reaches a maximum of 5.8 at $\% \mathrm{Ca}$ at $585^{\circ} \mathrm{C}$.

Table 1:

(Ca-Sb) Crystal Structure Data

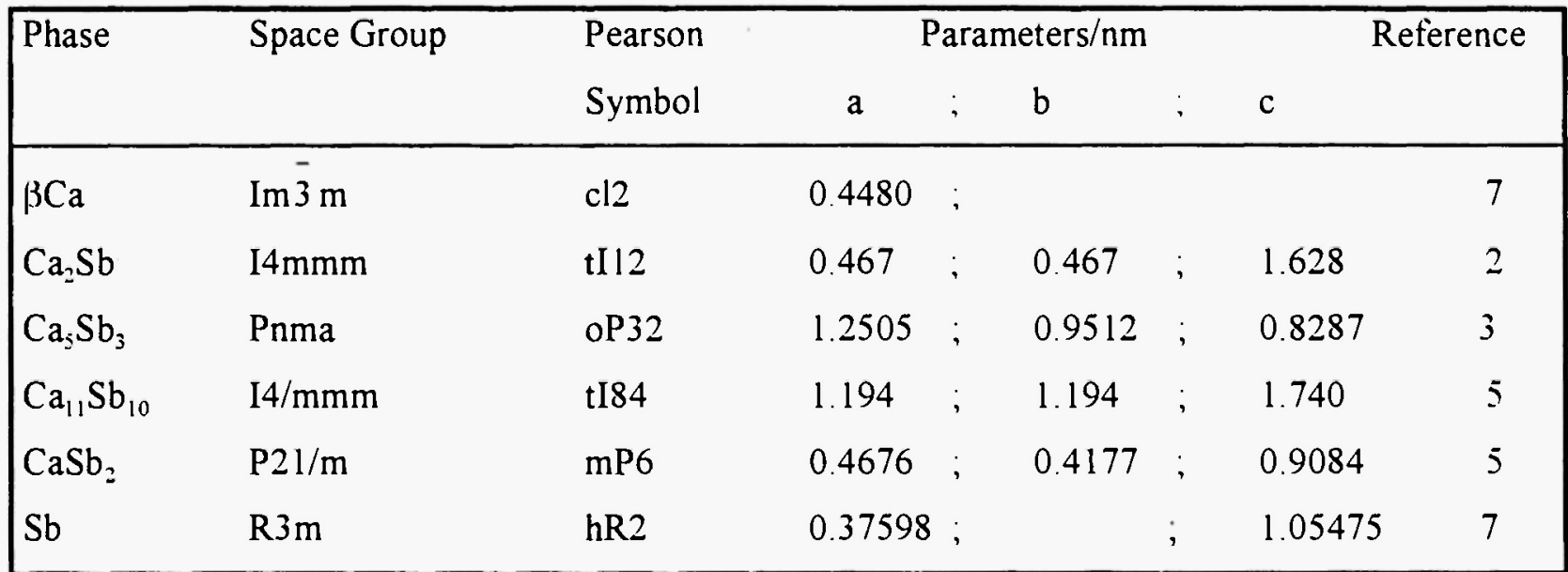




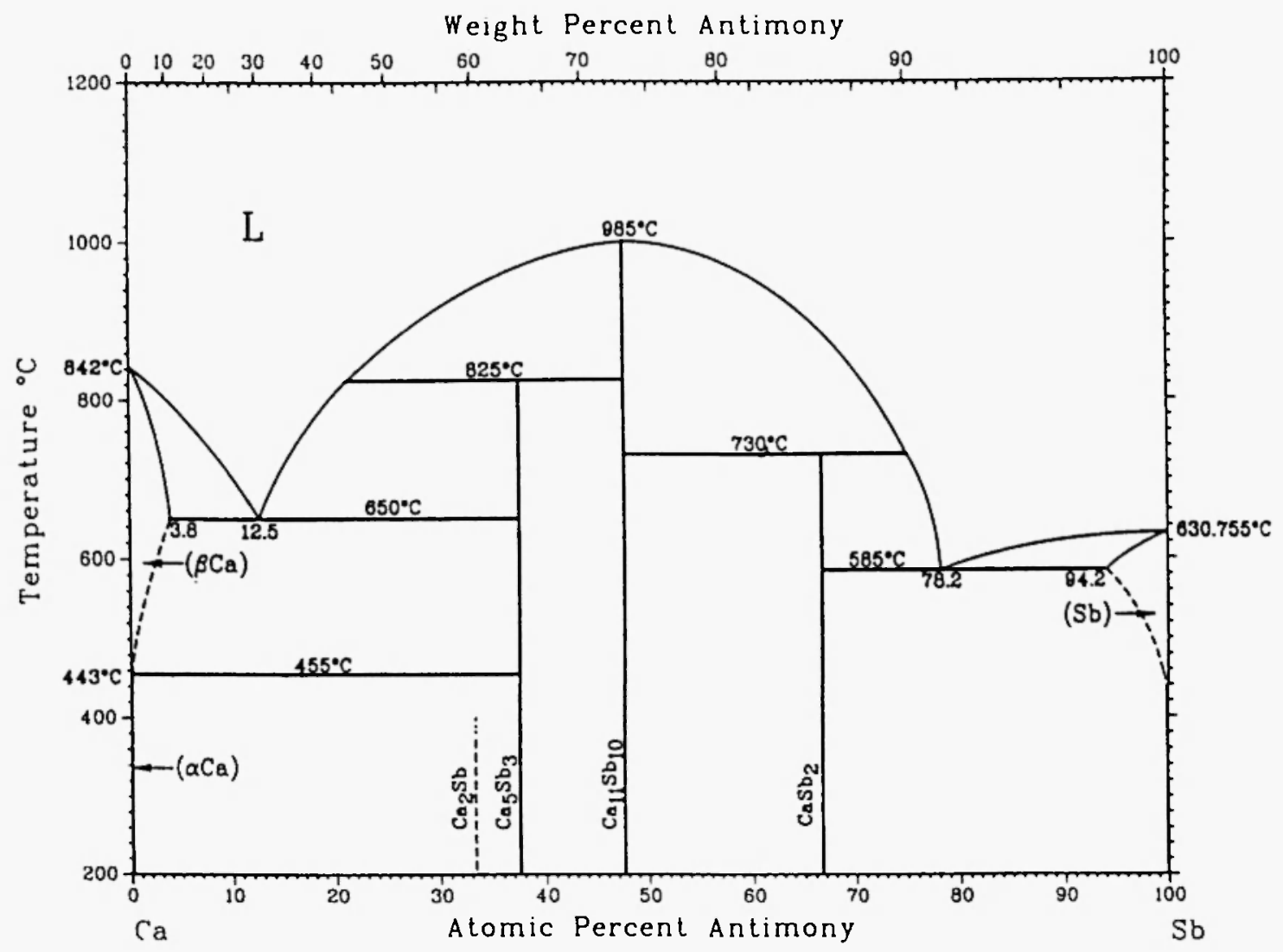

Fig 1: The $\mathrm{Ca}-\mathrm{Sb}$ phase diagram according to Massalski et al. $/ 6 /$.

As shown in Figure 1, the diagram compiled by Massalski et al. /6/ gives the same melting temperatures as given in the literature.

The present work reports the results of the $\mathrm{Ca}-\mathrm{Sb}$ system using differential thermal analysis (DTA) in the antimony rich part and calorimetric determinations of enthalpies of formation of liquid and of two phase alloys. A numerical optimisation technique is also presented.

\section{EXPERIMENTAL AND RESULTS}

Antimony and calcium were prepared from Ventron-Alfa products and their purities were 99.99 and $99.95 \%$ respectively. For (DTA) experiments, samples were prepared by two methods:

- For $\left(\mathrm{X}_{\mathrm{C}_{\mathrm{a}}} \leq 0.33\right)$ alloys, pure metals were put into a silica tube under vacuum and heated at $860^{\circ} \mathrm{C}$ during two days, then kept at $620^{\circ} \mathrm{C}$ during another two days and slowly cooled.

- For $\left(0.33<\mathrm{X}_{\mathrm{Ca}} \leq 0.67\right)$ alloys, pure metals were layed on an alumina crucible before they were put into the silica tube. The heating temperature was successively $1050^{\circ} \mathrm{C}$ and $800^{\circ} \mathrm{C}$ during two days. DTA used a SETARAM furnace with tungsten resistor; the sample and the alumina reference were set in an alumina crucible inside a pyrex tube under argon flow (Argon U; liquid air). The differential signal and temperature signal were obtained by means of Pt-(PtRh $10 \%$ ) thermocouples.

The calorimetric measurements were made using a Calvet calorimeter under argon flow near $800^{\circ} \mathrm{C}$. The calibration was carried out by dropping antimony shots, taken at room temperature, in the calorimeter; the calorimetric signal was tied with the melting and heating enthalpy. Calcium samples were prepared and weighed in a glove box; they were added to the 
antimony bath; the mean calcium partial enthalpy of mixing was obtained as well as the enthalpy of formation .

The results obtained in this work are summarised in Table 2 and the peak temperatures are given in Figure 2. For the equimolecular composition, the $\mathrm{Ca}-\mathrm{Sb}$ system appears to be often in a metastable state which may be attributed to local segregation. The $\mathrm{Ca}_{11} \mathrm{Sb}_{10}$ compound shows a high melting temperature $\left(1120{ }^{\circ} \mathrm{C}\right)$ which contrasts with the corresponding value of $985^{\circ} \mathrm{C}$ indicated by Niyazova et al. /1/.

The X-ray analysis was carried out in a Lindemann tube. However, it was difficult to obtain good spectra, so that the results were missing for several samples. On the other hand microprobe analysis has been performed on $\mathrm{Sb}$ rich samples. The samples $\left(\mathrm{X}_{\mathrm{Sb}}-0.90 ; 0.85\right.$;
$0.82 ; 0.78$ ) were prepared under vacuum in silica tube at $800^{\circ} \mathrm{C}$ for 2 hours, then maintained at $585^{\circ} \mathrm{C}$ for 5 hours and air-quenched. They were polished without any liquid. The (Sb) phase was found not to contain any $\mathrm{Ca}$ component, so that we could not detect the $\mathrm{CaSb}_{2}$ phase reported by Niyazova et al. /1/. The present results only suggest the existence of a Sb primary solid solution.

With respect to the eutectic composition of liquid, the $\mathrm{Sb}$ primary precipitate area and the eutectic precipitate area were tested by measuring the microphotograph of an alloy with $\mathrm{X}_{\mathrm{Ca}_{\mathrm{a}}}=\mathbf{0 . 8 5}$. It is quite likely that these areas are proportional to the volume of the precipitates. As the specific gravity of $\mathrm{Sb}$ and $\mathrm{CaSb}_{2}$ are 7.8 and $5.45 \mathrm{~g} \mathrm{~cm}^{-3}$ respectively, the eutectic composition is estimated to be $\mathrm{X}_{\mathrm{Ca}_{\mathrm{a}}}=0.78$.

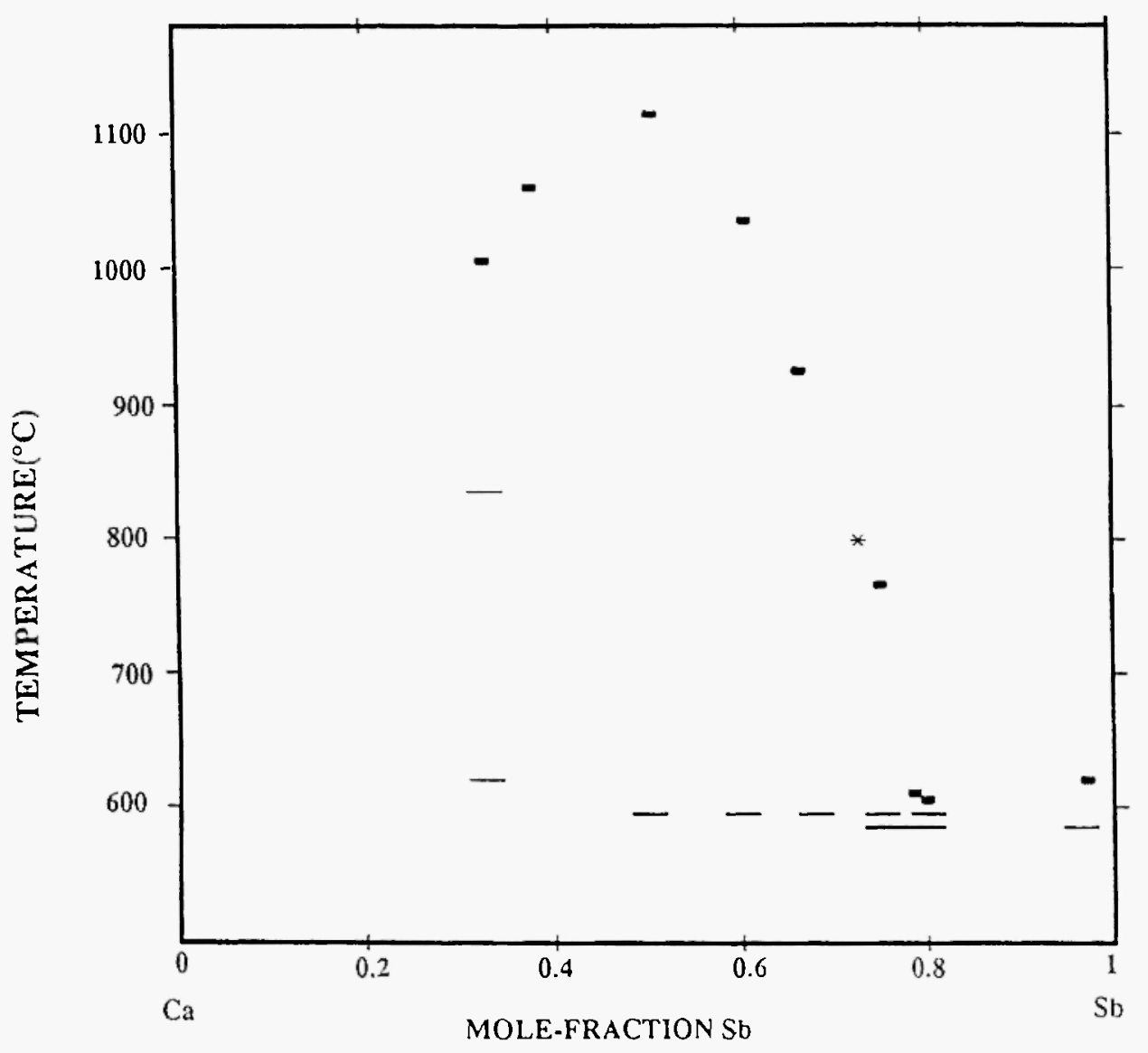

Fig 2: Experimental points of the $\mathrm{Ca}-\mathrm{Sb}$ diagram.

- Solidus points (DTA).

$==$ Liquidus points (DTA).

* Liquidus points (Calorimetry). 
Table 2

A: silica sealed ampoule (under vacuum) 2 days at $860^{\circ} \mathrm{C}, 2$ days at $620^{\circ} \mathrm{C}$ B: alumina crucible in silica sealed ampoule (under vacuum) 2 days at $1050^{\circ} \mathrm{C}, 2$ days at $800^{\circ} \mathrm{C}$ L: Liquid. $\uparrow$ : increasing temperature; $\downarrow$ : decreasing temperature.

\begin{tabular}{|c|c|c|c|c|c|c|}
\hline $\mathrm{X}_{\mathrm{si}, \%} \%$ & \multicolumn{2}{|c|}{ RX Analysis } & \multicolumn{2}{|c|}{ DTA Peak } & Interpretation & Preparation \\
\hline & Bctore DTA & After DTA & Out sct & Maximum & & \\
\hline $33 \uparrow$ & $\mathrm{Ca}+\mathrm{Ca}_{2} \mathrm{Sb}$ & & $\begin{array}{l}309^{\circ} \mathrm{C} \\
660^{\circ} \mathrm{C} \\
840^{\circ} \mathrm{C}\end{array}$ & $\begin{array}{l}420^{\circ} \mathrm{C} \\
1004^{\circ}\end{array}$ & $\begin{array}{l}? \alpha \text { and } \beta \mathrm{Ca} \text { transformation } \\
\text { Light peak } \beta \mathrm{Ca}+\mathrm{Ca} \mathrm{S}_{2} \mathrm{Sb} \Leftrightarrow \mathrm{L} \\
\mathrm{Ca}_{5} \mathrm{Sb}_{3} \Leftrightarrow \mathrm{Ca} \mathrm{C}_{3} \mathrm{Sb}+\mathrm{L} \\
\text { Liquidus line }\end{array}$ & B \\
\hline $\begin{array}{l}38 \uparrow \\
38 \downarrow\end{array}$ & & $\mathrm{Ca}_{5} \mathrm{Sb}_{3}+\mathrm{Ca}_{11} \mathrm{Sb}_{111}$ & $\begin{array}{l}877^{\circ} \mathrm{C} \\
825^{\circ} \mathrm{C} \\
606^{\circ} \mathrm{C} \\
554^{\circ} \mathrm{C} \\
\end{array}$ & $1059^{\circ} \mathrm{C}$ & $\begin{array}{l}\mathrm{Ca}_{5} \mathrm{Sb}_{3} \Leftrightarrow \mathrm{Ca}_{11} \mathrm{Sb}_{11}+\mathrm{L} \\
\text { Liquidus line }\end{array}$ & B \\
\hline $\begin{array}{l}50 \uparrow \\
50 \downarrow\end{array}$ & & & $\begin{array}{l}555^{\circ} \mathrm{C} \\
618^{\circ} \mathrm{C} \\
830^{\circ} \mathrm{C} \\
932^{\circ} \mathrm{C}\end{array}$ & & $\begin{array}{l}\text { metastable evolution } \\
\mathrm{CaSb}_{2}+\mathrm{Ca}_{11} \mathrm{Sb}_{11} \text { eutectic? } \\
\text { metastable evolution }\end{array}$ & B \\
\hline $50 \downarrow$ & & $\mathrm{CaSb}_{2}+\mathrm{Ca}_{11} \mathrm{Sb}_{10}$ & $\begin{array}{l}610^{\circ} \mathrm{C} \\
560^{\circ} \mathrm{C}\end{array}$ & & & B \\
\hline $50 \uparrow$ & & & $\begin{array}{l}567^{\circ} \mathrm{C} \\
606^{\circ} \mathrm{C} \\
\end{array}$ & & metastable evolution & B \\
\hline $50 \uparrow$ & & & $597^{\circ} \mathrm{C}$ & $1120^{\circ} \mathrm{C}$ & $\begin{array}{l}\mathrm{CaSb}_{2} \Leftrightarrow \mathrm{Ca}_{11} \mathrm{Sb}_{10}+\mathrm{L} \\
\text { Liquidus line }\end{array}$ & B \\
\hline $60 \uparrow$ & & & $585^{\circ} \mathrm{C}$ & $1034^{\circ} \mathrm{C}$ & $\begin{array}{l}\mathrm{CaSb}_{2} \Leftrightarrow \mathrm{Ca}_{11} \mathrm{Sb}_{11}+\mathrm{L} \\
\text { Liquidus line }\end{array}$ & B \\
\hline $60 \downarrow$ & & & & $\begin{array}{l}595^{\circ} \mathrm{C} \\
560^{\circ} \mathrm{C}\end{array}$ & & B \\
\hline $\begin{array}{l}67 \uparrow \\
67 \downarrow\end{array}$ & $\mathrm{CaSb}_{2}$ & $\mathrm{CaSb}_{2}$ & $\begin{array}{r}597^{\circ} \mathrm{C} \\
599^{\circ} \mathrm{C} \\
\end{array}$ & $923^{\circ} \mathrm{C}$ & $\begin{array}{l}\mathrm{CaSb}_{2} \Leftrightarrow \mathrm{Ca}_{11} \mathrm{Sb}_{10}+\mathrm{L} \\
\text { Liquidus line } \\
\mathrm{CaSb}_{2} \Leftrightarrow \mathrm{Ca}_{11} \mathrm{Sb}_{10}+\mathrm{L}\end{array}$ & $A$ \\
\hline $70 \uparrow$ & $(\mathrm{Sb})+\mathrm{CaSb}_{2}$ & $(\mathrm{Sb})+\mathrm{CaSb}_{2}$ & $\begin{array}{l}440^{\circ} \mathrm{C} \\
585^{\circ} \mathrm{C} \\
613^{\circ} \mathrm{C} \\
\end{array}$ & & $\begin{array}{l}(\mathrm{Sb})+\mathrm{CaSb}_{2} \Leftrightarrow \mathrm{L} \\
\mathrm{CaSb}_{2} \Leftrightarrow \mathrm{L} ? \text { (metatable) }\end{array}$ & $A$ \\
\hline $75 \downarrow$ & $(\mathrm{Sb})+\mathrm{CaSb}_{2}$ & $(\mathrm{Sb})+\mathrm{CaSb}_{2}$ & $\begin{array}{l}445^{\circ} \mathrm{C} \\
585^{\circ} \mathrm{C} \\
598^{\circ} \mathrm{C} \\
\\
626^{\circ} \mathrm{C} \\
598^{\circ} \mathrm{C} \\
\end{array}$ & $760^{\circ} \mathrm{C}$ & $\begin{array}{l}(\mathrm{Sb})+\mathrm{CaSb}_{2} \Leftrightarrow \mathrm{L} \\
\mathrm{CaSb}_{2} \Leftrightarrow \mathrm{Ca}_{11} \mathrm{Sb}_{10}+\mathrm{L} \\
\text { Liquidus line } \\
\mathrm{CaSb}_{2} \Leftrightarrow \mathrm{Ca}_{11} \mathrm{Sb}_{1,1}+\mathrm{L}\end{array}$ & $A$ \\
\hline $80 \uparrow$ & $(\mathrm{Sb})+\mathrm{CaSb}_{2}$ & $(\mathrm{Sb})+\mathrm{CaSb}_{2}$ & $\begin{array}{l}587^{\circ} \mathrm{C} \\
597^{\circ} \mathrm{C}\end{array}$ & $611^{\circ} \mathrm{C}$ & $\begin{array}{l}(\mathrm{Sb})+\mathrm{CaSb}_{2} \Leftrightarrow \mathrm{L} \\
\mathrm{CaSb}_{2} \Leftrightarrow \mathrm{Ca}_{11} \mathrm{Sb}_{11}+\mathrm{L} \\
\text { liquidus line }\end{array}$ & $A$ \\
\hline $\begin{array}{l}90 \uparrow \\
90 \downarrow\end{array}$ & $(\mathrm{Sb})+\mathrm{CaSb}_{2}$ & $(\mathrm{Sb})+\mathrm{CaSb}_{2}$ & $587^{\circ} \mathrm{C}$ & $\begin{array}{l}628^{\circ} \mathrm{C} \\
628^{\circ} \mathrm{C}\end{array}$ & $\begin{array}{l}(\mathrm{Sb})+\mathrm{CaSb}_{2} \Leftrightarrow \mathrm{L} \\
\text { liquidus line }\end{array}$ & $A$ \\
\hline
\end{tabular}




\section{ENTHALPY DETERMINATIONS}

The $\mathrm{Ca}$ shots in the Sb-liquid bath reacted vigorously for forming of liquid alloy. When $\mathrm{X}_{\mathrm{C}}$ increased, the reaction became slower. The calorimetric measurements were possible until $\mathrm{X}_{\mathrm{C}_{\mathrm{a}}}=0.60$. Three determinations were made at two temperatures $\left(704^{\circ} \mathrm{C}\right.$ and $750^{\circ} \mathrm{C}$ ). For alloy with the composition $\mathrm{X}_{\mathrm{C}}=0.39$, the $\mathrm{X}$-ray analysis indicated both $\mathrm{CaSb}_{2}$ and $\mathrm{Ca}_{11} \mathrm{Sb}_{10}$. For $\mathrm{X}_{\mathrm{Ca}}=0.60$, phases of $\mathrm{Ca}_{5} \mathrm{Sb}_{3}$ and $\mathrm{Ca}_{11} \mathrm{Sb}_{10}$ were suggested. On the other hand, for $\mathrm{X}_{\mathrm{C}_{\mathrm{a}}}=0.64$, the results showed the $\mathrm{Ca}_{5} \mathrm{Sb}_{3}$ and $\mathrm{Ca}_{2} \mathrm{Sb}$ structures. The results are summarised in Figure 3 and Table 3, providing information about the formation of liquid phase. These results suggest the linear domain of $\mathrm{Ca}_{11} \mathrm{Sb}_{10}$ inside the liquid phase and the linear domain of $\mathrm{Ca}_{5} \mathrm{Sb}_{3}$ arising from a reaction of $\mathrm{Ca}$ on $\mathrm{Ca}_{11} \mathrm{Sb}_{10}$. The present work also provides information about a point of liquidus line

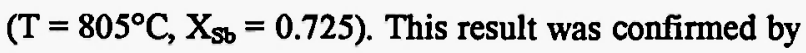
DTA.

In the liquid phase, the integral enthalpies of formation are given as follows:

$\underline{\mathrm{T}=704^{\circ} \mathrm{C}} \quad \Delta \mathrm{H}_{\mathrm{f}}\left(\mathrm{X}_{\mathrm{C}}=0.10\right)=-17 \mathrm{~kJ} / \mathrm{mol}$ of atoms. $\Delta \mathrm{H}_{\mathrm{f}}\left(\mathrm{X}_{\mathrm{Cs}}=0.20\right)=-33.6 \mathrm{~kJ} / \mathrm{mol}$ of atoms.

$\underline{\mathrm{T}=750^{\circ} \mathrm{C}} \quad \Delta \mathrm{H}_{\mathrm{f}}\left(\mathrm{X}_{\mathrm{Cs}}=0.10\right)=-19 \mathrm{~kJ} / \mathrm{mol}$ of atoms. $\Delta \mathrm{H}_{\mathrm{f}}\left(\mathrm{X}_{\mathrm{Cs}_{\mathrm{s}}}=0.20\right)=-36.5 \mathrm{~kJ} / \mathrm{mol}$ of atoms.

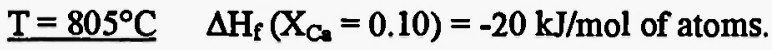
$\Delta \mathrm{H}_{\mathrm{f}}\left(\mathrm{X}_{\mathrm{Cs}}=0.20\right)=-39 \mathrm{~kJ} / \mathrm{mol}$ of atoms.

The minimum in the enthalpy curve, $-107 \mathrm{~kJ} / \mathrm{mol}$ of atoms, corresponds to the $\mathrm{Ca}_{11} \mathrm{Sb}_{10}\left(\mathrm{X}_{\mathrm{Sb}}=0.476\right)$ composition and we obtain the following result.

Table 3

Enthalpy of formation of (Ca-Sb) alloys at $805^{\circ} \mathrm{C}$

Calorimeter calibration:

Enthalpy changes during transformations:

$\mathrm{Sb}\left(\mathrm{S}, 32^{\circ} \mathrm{C}\right) \rightarrow \mathrm{Sb}\left(\mathrm{Liq}, 805^{\circ} \mathrm{C}\right)$ and $\mathrm{Ca}\left(\mathrm{S}, 32^{\circ} \mathrm{C}\right) \rightarrow \mathrm{Ca}\left(\mathrm{S}, 805^{\circ} \mathrm{C}\right)$

$\mathrm{H}\left(\mathrm{Sb}, \mathrm{Liq}, 805^{\circ} \mathrm{C}\right)-\mathrm{H}\left(\mathrm{Sb}, \mathrm{S}, 32^{\circ} \mathrm{C}\right)=3427 \mathrm{~J} / \mathrm{mol}$ of atoms.

$\mathrm{H}\left(\mathrm{Ca}, \mathrm{S}, 805^{\circ} \mathrm{C}\right)-\mathrm{H}\left(\mathrm{Ca}, \mathrm{S}, 32^{\circ} \mathrm{C}\right)=6163 \mathrm{~J} / \mathrm{mol}$ of atoms.

\begin{tabular}{llll}
\hline $100 \mathrm{X}_{\mathrm{ca}}$ & $\Delta \mathrm{H}_{\mathrm{f}}(\mathrm{kJ} / \mathrm{mol}$ atom $)$ & $100 \mathrm{X}_{\mathrm{iad}}$ & $\Delta \mathrm{H}_{\mathrm{i}}(\mathrm{kJ} / \mathrm{mol}$ atom $)$ \\
\hline 04.26 & 08.18 & 42.86 & 84.88 \\
06.31 & 12.68 & 45.52 & 92.30 \\
10.71 & 20.41 & 46.20 & 95.00 \\
13.95 & 23.62 & 47.34 & 97.50 \\
16.04 & 27.50 & 49.90 & 102.50 \\
20.34 & 32.21 & 53.25 & 106.29 \\
23.22 & 37.54 & 54.52 & 105.94 \\
26.19 & 43.25 & 55.97 & 103.37 \\
28.78 & 48.32 & 57.31 & 101.08 \\
31.70 & 55.30 & 58.30 & 99.30 \\
34.94 & 63.68 & 59.23 & 97.28 \\
37.19 & 71.40 & 60.07 & 95.44 \\
40.63 & 78.76 & &
\end{tabular}


$\Delta \mathrm{H}_{\mathrm{f}}\left(1 / 21 \mathrm{Ca}_{11} \mathrm{Sb}_{10} ; 805^{\circ} \mathrm{C}\right)=-(107 \pm 3) \mathrm{kJ} / \mathrm{mol}$ of atoms (referred to solid $\mathrm{Ca}$ and liquid $\mathrm{Sb}$ ).

The enthalpy of formation of $\mathrm{Ca}_{5} \mathrm{Sb}_{3}$ was also obtained by extrapolation of the enthalpy curve for $X_{C a}>0.528$ until $\mathrm{X}_{\mathrm{Ca}}=0.625$.

$\Delta \mathrm{H}_{f}\left(1 / 8 \mathrm{Ca}_{5} \mathrm{Sb}_{3} ; 805^{\circ} \mathrm{C}\right)=-(86 \pm 6) \mathrm{kJ} / \mathrm{mol}$ of atoms. (referred to solid $\mathrm{Ca}$ and liquid $\mathrm{Sb}$ ).

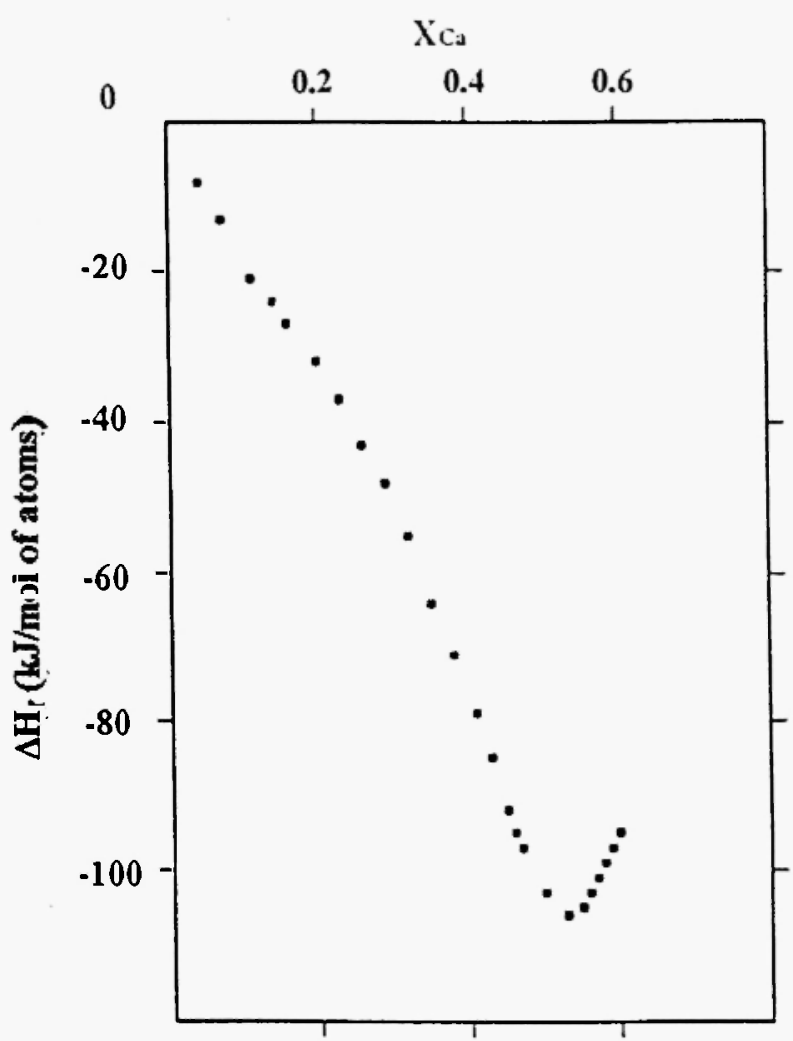

Fig 3: Enthalpy of formation of the (Ca-Sb) alloys at $805^{\circ} \mathrm{C}$.

\section{REFINEMENT OF THE Ca-Sb SYSTEM USING LUCKAS PROGRAM}

This program consisting of three principal subroutines has been written by Luckas and his group 17/; it allows to compute and to plot the phase diagram and the thermodynamic functions of binary systems.
This technique supplements the experimental data. The BINGSS program $/ 7 /$ is a least square fitting program for binary system, using simultaneously phase diagram and thermodynamic data.

The program of BINFKAT /7/ uses polynomial descriptions for the excess terms of the Gibbs energy and it is used to calculate the phase diagram and draw thermodynamic functions for testing the results of BINGSS 171 . Finally with a plot program $n 1$, the experimental data which are inputs to BINGSS $/ 7 /$ can be plotted together with the calculated curves.

\section{1. Input Of Experimental Values}

\begin{tabular}{|c|c|c|}
\hline Invariant: $\mathrm{Sb}_{-} \mathrm{Sb}_{2}$ & $\begin{array}{c}\text { Temperature: } \\
587^{\circ} \mathrm{C}\end{array}$ & $X_{S b}($ liquid $)=0.82$ \\
\hline $\begin{array}{l}\text { Invariant: } \\
\mathrm{CaSb}_{2}-\mathrm{Ca}_{11} \mathrm{Sb}_{10}\end{array}$ & $\begin{array}{c}\text { Temperature: } \\
597^{\circ} \mathrm{C} \\
\end{array}$ & $\mathrm{X}_{\mathrm{Sb}}($ liquid $)=0.79$ \\
\hline $\begin{array}{l}\text { Invariant: } \\
\mathrm{Ca}-\mathrm{Ca}_{2} \mathrm{Sb}\end{array}$ & $\begin{array}{c}\text { Temperature: } \\
620^{\circ} \mathrm{C} \\
\end{array}$ & $\begin{array}{c}X_{\mathrm{Sb}}(\text { liquid })= \\
0.125\end{array}$ \\
\hline $\begin{array}{c}\text { Invariant: } \\
\mathrm{Ca}_{2} \mathrm{Sb}_{-} \mathrm{Ca}_{5} \mathrm{Sb}_{3}\end{array}$ & $\begin{array}{c}\text { Temperature: } 840^{\circ} \\
\mathrm{C} \\
\end{array}$ & $\mathrm{X}_{\mathrm{Sb}}$ (liquid) $=0.22$ \\
\hline $\begin{array}{l}\text { Equilibrium: } \\
\text { Liquid-Sb }\end{array}$ & $\begin{array}{c}\text { Temperature: } \\
628^{\circ} \mathrm{C}\end{array}$ & $\mathrm{X}_{\mathrm{Sb}}$ (liquid) $=0.90$ \\
\hline \multirow[t]{6}{*}{$\begin{array}{c}\text { Equilibrium: } \\
\text { Liquid-Ca }{ }_{11} \mathrm{Sb}_{10}\end{array}$} & Temperature $\left({ }^{\circ} \mathrm{C}\right)$ & $\mathrm{X}_{\mathrm{Sb}}$ (liquid) \\
\hline & 805 & 0.73 \\
\hline & 923 & 0.67 \\
\hline & 1034 & 0.50 \\
\hline & 1050 & 0.38 \\
\hline & 1004 & 0.325 \\
\hline
\end{tabular}

Enthalpies of formation ( $\mathrm{J} / \mathrm{mol}$ of atoms) at temperature: $805^{\circ} \mathrm{C}$

$\begin{array}{lll}\mathrm{Ca}_{11} \mathrm{Sb}_{10} & -107000 & \\ \mathrm{Ca}_{5} \mathrm{Sb}_{3} & -86000 & \\ \text { Liquid } & -8200 & \mathrm{X}_{\mathrm{Sb}}=0.958 \\ & -20400 & \mathrm{X}_{\mathrm{Sb}}=0.893 \\ & -27500 & \mathrm{X}_{\mathrm{Sb}}=0.840 \\ & -37500 & \mathrm{X}_{\mathrm{Sb}}=0.768 \\ & -43200 & \mathrm{X}_{\mathrm{Sb}}=0.738\end{array}$

(referred to solid $\mathrm{Ca}$ and liquid $\mathrm{Sb}$ ) 


\section{2. Output Values From Computation}

\begin{tabular}{|c|c|c|}
\hline $\begin{array}{l}\text { Invariant: } \\
\text { Sb-CaSb }\end{array}$ & $\begin{array}{c}\text { Temperature: } \\
587^{\circ} \mathrm{C}\end{array}$ & $\mathrm{X}_{\mathrm{Sb}}$ (liquid) $=0.82$ \\
\hline $\begin{array}{l}\text { Invariant: } \\
\mathrm{CaSb}_{2}-\mathrm{Ca}_{11} \mathrm{Sb}_{10}\end{array}$ & $\begin{array}{c}\text { Temperature: } \\
597^{\circ} \mathrm{C}\end{array}$ & $\mathrm{X}_{\mathrm{Sb}}$ (liquid) $=0.79$ \\
\hline $\begin{array}{l}\text { Invariant: } \\
\mathrm{Ca}_{-}-\mathrm{Ca}_{2} \mathrm{Sb}\end{array}$ & $\begin{array}{c}\text { Temperature: } \\
619^{\circ} \mathrm{C} \\
\end{array}$ & $\begin{array}{c}\mathrm{X}_{\mathrm{Sb}}(\text { liquid })= \\
0.182\end{array}$ \\
\hline $\begin{array}{l}\text { Invariant: } \\
\mathrm{Ca}_{2} \mathrm{Sb}_{-} \mathrm{Ca}_{5} \mathrm{Sb}_{3}\end{array}$ & $\begin{array}{c}\text { Temperature: } \\
840^{\circ} \mathrm{C} \\
\end{array}$ & $\mathrm{X}_{\text {St }}($ liquid $)=0.22$ \\
\hline $\begin{array}{l}\text { Invariant: } \\
\mathrm{Ca}_{11} \mathrm{Sb}_{10}-\mathrm{Ca}_{5} \mathrm{Sb}_{3}\end{array}$ & $\begin{array}{c}\text { Temperature: } \\
1010^{\circ} \mathrm{C} \\
\end{array}$ & $\mathrm{X}_{\mathrm{Sb}}($ liquid $)=0.34$ \\
\hline $\begin{array}{l}\text { Equilibrium: } \\
\text { Liquid-Sb }\end{array}$ & $\begin{array}{c}\text { Temperature: } \\
625^{\circ} \mathrm{C}\end{array}$ & \\
\hline \multicolumn{3}{|l|}{$X_{S b}($ liquid $)=0.90$} \\
\hline \multirow[t]{7}{*}{$\begin{array}{l}\text { Equilibrium: } \\
\text { Liquid-Ca } \mathrm{a}_{11} \mathrm{Sb}_{10} \text { : }\end{array}$} & Temperature $\left({ }^{\circ} \mathrm{C}\right)$ & $\mathrm{X}_{\mathrm{Sb}}$ (liquid) \\
\hline & 805 & 0.71 \\
\hline & 923 & 0.66 \\
\hline & 800 & 0.60 \\
\hline & 1120 & 0.51 \\
\hline & 1050 & 0.36 \\
\hline & 1004 & 0.335 \\
\hline
\end{tabular}

Enthalpies of formation (J/mol of atoms) at $805^{\circ} \mathrm{C}$ referred to solid $\mathrm{Ca}$ and liquid $\mathrm{Sb}$.

$$
\begin{array}{lll}
\mathrm{Ca}_{11} \mathrm{Sb}_{10}: & -109000 & \\
\mathrm{Ca}_{5} \mathrm{Sb}_{3:} & -86000 & \\
\text { Liquid: } & -7880 & \mathrm{X}_{\mathrm{Sb}}=0.95 \\
& -16000 & \mathrm{X}_{\mathrm{Sb}}=0.90 \\
& -25120 & \mathrm{X}_{\mathrm{Sb}}=0.85 \\
& -33600 & \mathrm{X}_{\mathrm{Sb}}=0.80 \\
& -40500 & \mathrm{X}_{\mathrm{Sb}}=0.75
\end{array}
$$

\section{CONCLUSION}

The tracing (Figure 2) of a binary (Ca-Sb) system obtained from the present DTA and calorimetric measurements, suggests four stoichiometric compounds $\mathrm{Ca}_{2} \mathrm{Sb}, \mathrm{Ca}_{11} \mathrm{Sb}_{10}, \mathrm{Ca}_{5} \mathrm{Sb}_{3}$ and $\mathrm{CaSb}_{2}$. This is consistent with the results recently compiled by Massalski et al. 16/. The differential thermal analysis enables us to give some points of diagram lines with sufficient reliability. The peritectic melting of $\mathrm{CaSb}_{2}$ has been obtained in this work at $597^{\circ} \mathrm{C}$.

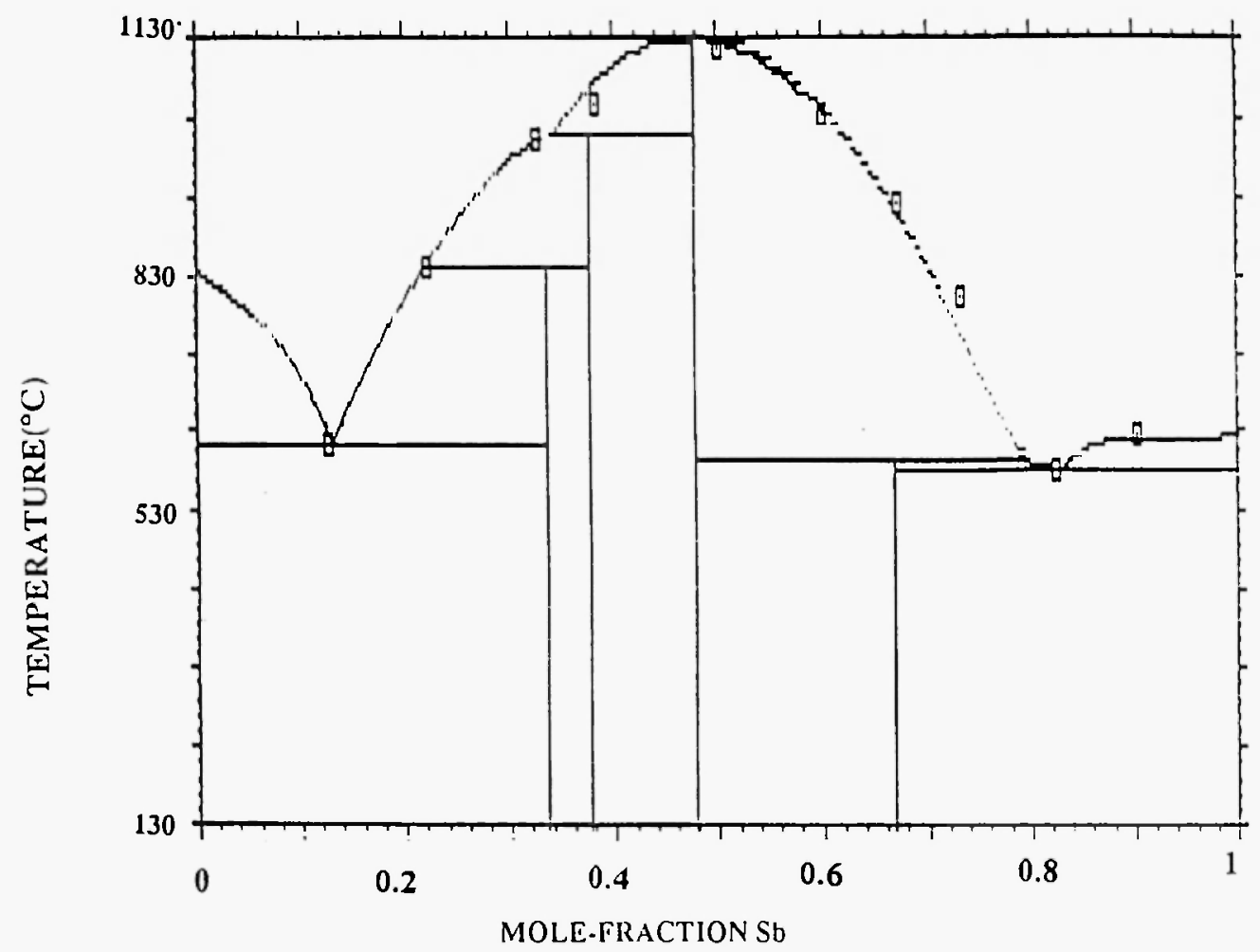

Fig 4: $\mathrm{Ca}-\mathrm{Sb}$ revised phase diagram. 


\section{REFERENCES}

1. Z. U. Niyazova, A.V. Vakhobov and T. D. Dzhuraev, Russ. Inorg. Mater., 12, 1074 (1976).

2. B. Eisenmann and H. Schäfer, Z. Naturforsch, B29, 13 (1974)

3. M. Martinez-Ripoll and G. Brauer, Acta Cryst., B30, 1083 (1961).

4. G. Brauer and O. Muller, Angew. Chem., 73, 169 (1961).

5. K. Deller and B. Eisenmann, Z. Naturforsch., B31, 29 (1976).

6. T. B. Massalski, H. Okamoto, P. R Subramanian and L. Kacprzak, Binary Alloy Phase Diagram, Second Edition, Vol. 1, ASM International., Materials Park. OH. 1990.

7. H. L. Lukas, E. T. Henig and B. Zimmermann, Calphad. 225, 1 (1977). 